\title{
Automatic Registration and Superimposed Display of CAG and Bull's Eye Map of Myocardial SPECT
}

\author{
R Haraguchi $^{1}$, N Sugimoto ${ }^{1}$, S Eiho $^{1}$, Y Ishida $^{2}$ \\ ${ }^{1}$ Graduate School of Informatics, Kyoto University, Kyoto, Japan \\ ${ }^{2}$ Department of Radiology, National Cardiovascular Center Hospital, Osaka, Japan
}

\begin{abstract}
We have reported[1] at the former CiC conference about a method of superimposing a coronary arterial tree on a bull's eye map of SPECT. In the method, the left ventricular epicardial surface model (LV surface model) was constructed from the SPECT data. Coronary images corresponding to the bull's eye map was obtained by mapping bi-plane coronary angiograms (CAG) onto the LV surface model. This paper reports that we improve the matching degree and apply the Powell method for parameters estimation. Furthermore, we improve the procedure for generating resultant images. These improvements enable us to automate several processes that required interactive procedures in the former work. We validated the improved method by numerical experiments with artificial data set and applied the method to clinical data set successfully.
\end{abstract}

\section{Introduction}

At the former $\mathrm{CiC}$ conference, we have introduced CAT's eye image[1]. That is a coronary arterial tree image superimposed on a bull's eye map of SPECT, as the integrated display of two kinds of information; shape of the coronary and blood perfusion in the myocardium. The CAT's eye image is easily obtainable through some manual pointing on CAG followed by an automatic matching process on bull's eye map. CAT's eye image enables us to grasp the myocardial function more accurately and easily in accordance with the coronary artery.

In order to obtain CAT's eye image, we need to estimate some parameters for registration between CAG and SPECT. In the previous report, we defined the matching degree and found the optimal parameters. But there were some problems: (1) It was necessary to set initial parameters manually to those close enough true ones, and (2) the registration process required long computation time.

This paper reports that we improve the matching degree and apply the Powell method for parameters estimation. Furthermore, we improve the procedure for generating resultant image. These improvements enable us to automate several processes that required interactive procedures in the former work.

\section{Subject data}

Subject data are the same as the ones in the former report[1]. ${ }^{99 m}$ Tc-tetrofosmin SPECT images were taken. We use re-sampled short-axis data. The matrix size is $64 \times 64$ pixels $\times$ about 30 slices. And CAG images of the same patient were also obtained. The size is $512 \times 512$ pixels $\times 8$ bits. We use end-diastolic (ED) phase images.

\section{Methods}

Our method is performed by using the following facts[1]: (1) Left coronary arteries are running on the left ventricular epicardial surface. (2) On a short-axis plane of SPECT image, the left ventricular epicardial surface can be assumed to be a circle. (3) LAD runs along the myocardial anterior groove, and LCX runs along the coronary sulcus. (4) Spatial resolution of SPECT image is far lower than that of CAG, so precise registration is not necessary.

In our method, we use a left ventricular epicardial surface model (LV surface model) that is constructed from the SPECT data (We will explain the details in Sec.3.2.2).

\subsection{Mathematical expression for registration of $L V$ surface model and CAG images}

The orientation of the left ventricular long-axis is represented by rotation angles of $\phi_{x}, \phi_{y}, \phi_{z}$ as Fig.1. The origin is set on the midpoint of the long-axis.

We use bi-plane CAG system which has frontal (Omegaarm) and lateral (C-arm) systems as shown in Fig.2. We obtain two projected CAG images $A_{f}$ and $A_{l}$.

Any three dimensional point $s=(x, y, z)$ on LV surface model and the 2D points $\boldsymbol{a}_{f}=\left(u_{f}, v_{f}\right)$ and $\boldsymbol{a}_{l}=\left(u_{l}, v_{l}\right)$ on $A_{f}$ and $A_{l}$ respectively are described by the following equations on the assumption of parallel projection [2]. 


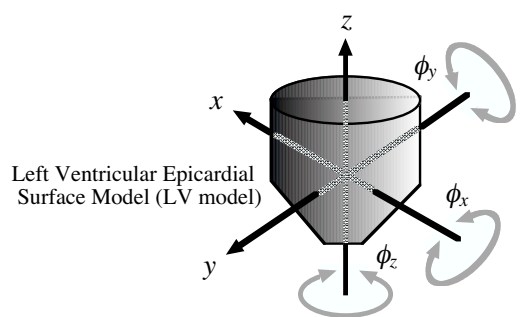

Figure 1. The orientation of LV surface model represented by rotation angles of three axes.

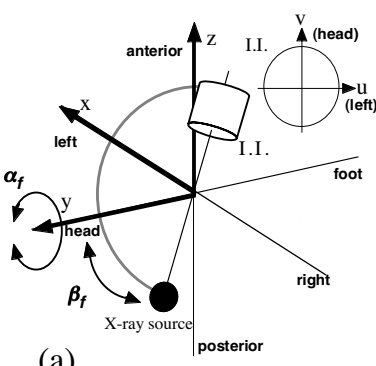

(a)

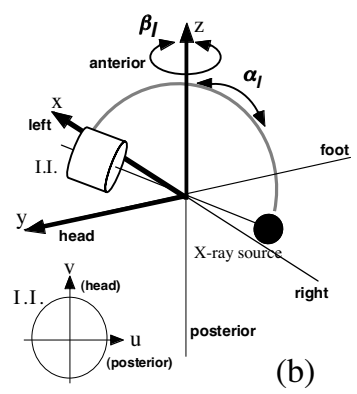

(b)
Figure 2. The angulation of bi-plane imaging system (a) The frontal system (b) The lateral system

For the frontal system

$\boldsymbol{a}_{f}=\mathrm{PR}_{x}\left(\beta_{f}\right) \mathrm{R}_{y}\left(\alpha_{f}\right) \mathbf{R}_{x}\left(\phi_{x}\right) \mathrm{R}_{y}\left(\phi_{y}\right) \mathbf{R}_{z}\left(\phi_{z}\right) m \boldsymbol{s}+\boldsymbol{c}_{f}$

For the lateral system

$\boldsymbol{a}_{l}=\mathrm{PR}_{y}\left(\alpha_{l}\right) \mathrm{R}_{z}\left(\beta_{l}\right) \mathrm{R}_{x}\left(\phi_{x}\right) \mathrm{R}_{y}\left(\phi_{y}\right) \mathbf{R}_{z}\left(\phi_{z}\right) m \boldsymbol{s}+\boldsymbol{c}_{l}$

where

$\mathbf{R}_{x}, \mathbf{R}_{y}, \mathbf{R}_{z}: 4 \times 4$ matrix represents $\mathrm{x}-, \mathrm{y}-, \mathrm{z}$-axis rotation

$\mathrm{P}$ : matrix represents parallel projection

$\alpha_{f}, \alpha_{l}$ : rotation toward left-right direction

$\beta_{f}, \beta_{l}$ : rotation toward head-foot direction

$m$ : scale parameter between SPECT and CAG image

$\boldsymbol{c}_{f}=\left(\Delta u_{f}, \Delta v_{f}\right), \boldsymbol{c}_{l}=\left(\Delta u_{l}, \Delta v_{l}\right):$ translation on each

\section{CAG image}

Equations (1)(2) contain 12 parameters in total. We define the registration parameter $\boldsymbol{p}$ as in Eq.(3).

$$
\begin{aligned}
\boldsymbol{p} & =\left(\boldsymbol{p}_{i}, \boldsymbol{p}_{p}\right) \\
\boldsymbol{p}_{i} & =\left(m, \alpha_{f}, \alpha_{l}, \beta_{f}, \beta_{l}\right) \\
\boldsymbol{p}_{p} & =\left(\phi_{x}, \phi_{y}, \phi_{z}, \Delta u_{f}, \Delta v_{f}, \Delta u_{l}, \Delta v_{l}\right)
\end{aligned}
$$

In this paper, we call $\boldsymbol{p}_{i}$ as a imaging vector. Imaging vector is approximately known from CAG device configuration. And we call $\boldsymbol{p}_{p}$ as a positioning vector. It is unknown vector and should be estimated.
The variable " $\phi_{z} "$ is corresponding to " $\theta$ " in the former report[1], which represents rotation of the resultant coronary image. We set it manually in the former. But in this report we will estimate it.

\subsection{Registration process}

\subsubsection{Pre-process}

The CAG images are enhanced their contrast by subtracting images at pre-injection. In the SPECT data, the rough positions of the anterior groove and coronary sulcus are set manually on one of the short-axis plane [1].

\subsubsection{LV surface model}

The LV surface model (Fig.1) is obtained from the SPECT data. We use the simple method of circle fitting calculated by Hough transformation on a short-axis plane[1]. The coordinates of the point $s=(x, y, z)$ on the LV surface model could be represented by two parameters. The first $r$ is the slice position $\left(0 \leq r \leq r_{\max }\right)$. And the second $\theta$ is the angle $\left(-180^{\circ} \leq \theta \leq 180^{\circ}\right)$. On the LV surface model, left coronary arteries distribute in the limited angle of $-165^{\circ} \leq \theta \leq 65^{\circ}[3]$. We call the area in this limited angle as LC-Area.

\subsubsection{Mapping CAG image onto the LV surface model}

If the vector $\boldsymbol{p}$ is given, each point $\boldsymbol{a}_{f}, \boldsymbol{a}_{l}$ could be corresponding to a point $s$ based on Eq.(1)(2). Then, CAG image $A_{f}$ can be mapped onto the LV surface model as shown in Fig.3(a). The mapping is performed only in the LC-Area.

We can describe thus mapped CAG image as $B_{f}(r, \theta ; \boldsymbol{p})$. Similarly we can obtain $B_{l}(r, \theta ; \boldsymbol{p})$ from $A_{l}$.

When in mapping procedure, some points can be mapped twice within the LC-Area, the other points are mapped only once within the LC-Area, as shown in Fig.3(a). In case of twice-mapping, pseudo image would appear in $B_{f}, B_{l}$. However, in case of once-mapping, pseudo image would not appear.
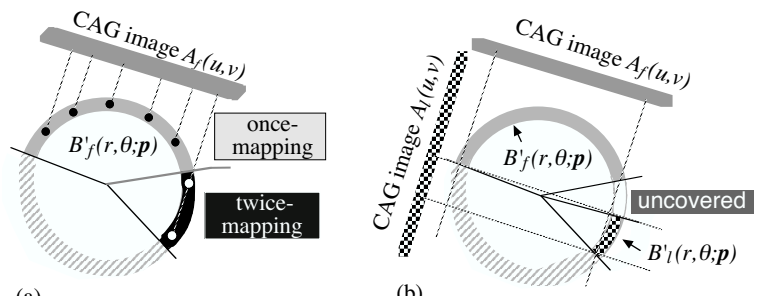

(a)

(b)

Figure 3. Mapping CAG images onto a LV surface model (viewing short-axis plane) (a) Mapping a CAG image once or twice (b) Mapping two CAG images and obtaining a resultant image 


\subsubsection{Automatic parameters estimation}

If the vector $\boldsymbol{p}$ is correct, two mapped CAG images $B_{f}$ and $B_{l}$ on the LV surface model should be the same, except for pseudo parts. Therefore, we estimate the vector $\boldsymbol{p}$ by maximizing a matching degree $J(\boldsymbol{p})$ between $B_{f}$ and $B_{l}$. In this paper, we define the matching degree as the multiplication of $B_{f}, B_{l}$ and weight $W$. (In this paper, a coronary part takes high value in CAG image.)

$$
J(\boldsymbol{p})=\sum_{r, \theta} B_{f}(r, \theta ; \boldsymbol{p}) \times B_{l}(r, \theta ; \boldsymbol{p}) \times W(r, \theta)
$$

We set high value for $W(r, \theta)$ in the positions of the anterior groove and the coronary sulcus. When LAD and LCX appear in such positions, $J(\boldsymbol{p})$ increases.

The vector $\boldsymbol{p}$ consists of $\boldsymbol{p}_{i}$ and $\boldsymbol{p}_{p}$. The vector $\boldsymbol{p}_{i}$ are approximately known from device's configuration, but the vector $\boldsymbol{p}_{p}$ are unknown. We initially set approximate values of $\boldsymbol{p}_{p}$ interactively assisted by GUI based program[2]. Next the correction of parameters error is done automatically by searching the maximum value of $J(\boldsymbol{p})$. In this paper, we use Powell method for maximizing $J(\boldsymbol{p})$.

In the previous work $[1,2]$, it was necessary to set the parameter $\phi_{z}$ interactively. However, by introducing the weight term $W$, the parameter $\phi_{z}$ could be also estimated automatically.

\subsubsection{Obtaining superimposed image}

The mapped image $B_{f}$ could be separated into two regions. One is once-mapping, and the other is twicemapping region. The former region has no pseudo part. The latter region may have pseudo part. Then we take only the once-mapping region from $B_{f}$. We call it $B_{f}^{\prime}$. Similarly, we obtain thus taken region $B_{l}^{\prime}$ from $B_{l}$ (Fig.3).

In almost all combinations of clinically common angles of bi-plane CAG system (such as LAO60 and RAO30), the joined region of $B_{f}^{\prime}$ and $B_{l}^{\prime}$ would cover almost all the LCArea. Then we can obtain a resultant image that consists of $B_{f}^{\prime}$ and $B_{l}^{\prime}$.

However, according to the projection angle of $\mathrm{CAG}$, over-lapped region of $B_{f}^{\prime}$ and $B_{l}^{\prime}$, or uncovered region by them, will appear in the LC-Area. We put a geometric mean value $\left(\sqrt{B_{f}(r, \theta) \times B_{l}(r, \theta)}\right)$ into such a region. By using a geometric mean procedure, pseudo part would be reduced. As a result, we can obtain the mapped coronary image $B(r, \theta)$ on the LV surface model.

By superimposing $B(r, \theta)$ on the same patient's SPECT image, we can obtain the integrated image of coronary anatomy and myocardial function. We can show it on 2D bull's eye map of SPECT and on 3-D LV surface model.

The procedure described here is newly added to our previous work $[1,2]$. In the previous work, we avoided pseudo part by manual selection.

\section{4. $\quad$ Results}

We validated the method proposed here by numerical experiments with artificial data set. An artificial LV surface model and artificial CAG images were used for the experiments. We represent true parameter as $\boldsymbol{p}_{\text {true }}$.

First, we investigated the characteristics of matching degree $J(\boldsymbol{p})$. Figure 4 shows the results when one element of the positioning vector $\boldsymbol{p}_{p}$ is changed respectively. Vertical axis represents matching degree ratio $J(\boldsymbol{p}) / J\left(\boldsymbol{p}_{\text {true }}\right)$ and horizontal axis represents parameter's deviation from $\boldsymbol{p}_{\text {true }}$. The amount per unit in horizontal axis is defined as an equivalent amount of nuclear image's voxelsize. Figure 5 shows the comparison of the improved matching degree with the former one. Figure 6 shows the results when multiple elements of the $\boldsymbol{p}_{p}$ are changed. Horizontal axis represents the distance from $\boldsymbol{p}_{\text {true }}$. There are several combinations of parameters having the same distance. The matching degree corresponding to is calculated on these combinations of parameters and is summarized average value $\left(J_{\text {ave }}\right)$ and maximum value $\left(J_{\max }\right)$.

Our method could be successfully applied to clinical cases. Fig.7(a) shows $B(r, \theta)$ in orthogonal map and Fig7(b) shows it in polar map. Figure 7(c) is a superimposed image, which is made from bull's eye map of SPECT and manually binarized Fig.7(b) image. Figure 7(d) shows a 3-D superimposed image on the LV surface model.
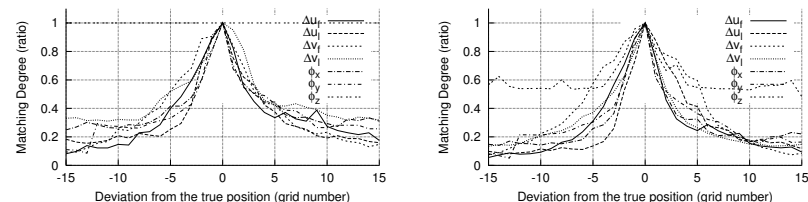

Figure 4. Characteristics of matching degree, changing one element of the positioning vector, (left)former one, (right)present one
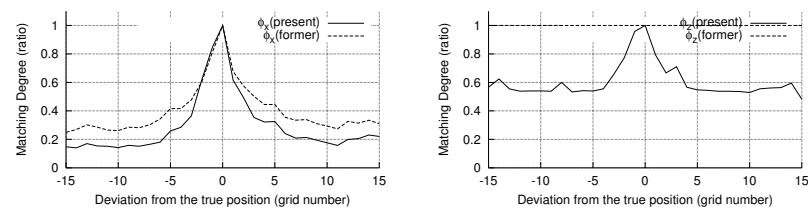

Figure 5. Characteristics of matching degree, comparing the former/present one (left) $\phi_{x}$, (right) $\phi_{z}$ 

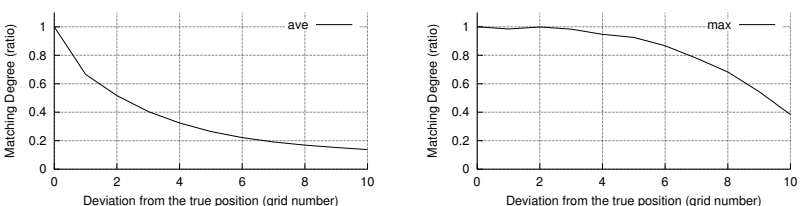

Figure 6. Characteristics of matching degree, changing multiple elements, (left) average (right) maximum

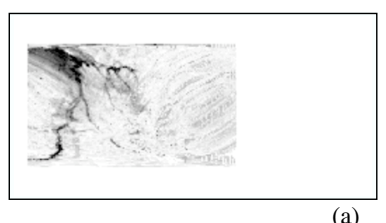

(a)

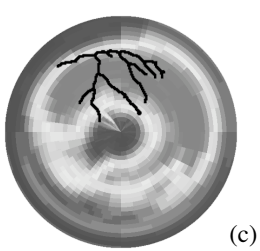

(c)

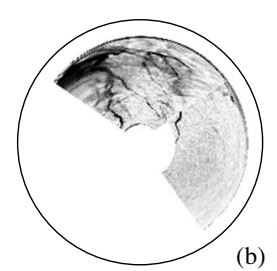

(b)

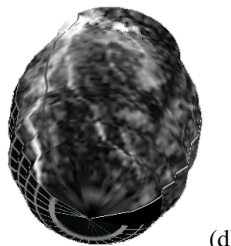

(d)
Figure 7. Results:

(a) mapped coronary image (orthogonal map), (b) mapped coronary image (polar map), (c) superimposed image on bull's eye map of SPECT, (d) superimposed image on LV surface model

\section{Discussion}

The effect of the weight term $W(r, \theta)$ into $J(\boldsymbol{p})$ is as follows: As shown in Fig.5(left), the characteristics of $J(\boldsymbol{p})$ is slightly improved. In the previous paper[1, 2], it was necessary to set the parameter $\phi_{z}(\theta$ in the former work) interactively. However, in this paper, $\phi_{z}$ can be estimated automatically as shown in Fig.5(right) because of introducing $W(r, \theta)$.

Figure 6 shows that, as a overall tendency, $J(\boldsymbol{p})$ monotonically decreases as deviation from the true position. Because of these good characteristics, we consider that we can estimate the optimal vector $\boldsymbol{p}$ automatically.

In the previous work $[1,2]$, we searched all points around the initial parameter set to find the optimal parameters which maximize the matching degree. In order to estimate the optimal parameters with practical computation time, it was necessary to set appropriate initial parameters manually. In this paper, we introduce Powell method for maximization. As a result, the computation time is reduced and the restriction on initial parameters is eased.

In order to generate a 3-D coronary image, it is usually necessary to identify feature points and their correspondence in CAG images [4]. However we need not perform those procedures and we can obtain a 3-D superimposed image in our method.

\section{Conclusion}

We improve our method of superimposing a coronary arterial tree on a bull's eye map of SPECT; 1) improve the matching degree and apply the Powell method for parameter estimation, 2) improve the method for generating a resultant image. These improvements enable us to automate several processes that require interactive procedures in the former work. And the restrictions on initial parameters were eased. We validated the method by numerical experiments and applied to clinical data set. The obtained images, superimposed images on a bull's eye map and on a 3-D LV surface model, give us clear understanding of the relationship between coronary artery and myocardium.

Now we are trying to extend our method to right coronary arterial tree and to integrate stenotic information on the resultant image.

\section{Acknowledgements}

This research is partially supported by Grant-in-Aid for Scientific Research (C)(2)(No.13680935) from Japan Society for the Promotion of Science(JSPS).

\section{References}

[1] Haraguchi R, Sugimoto N, Eiho S, Ishida Y. Cat's eye image : Superimposed display of the coronary artery on bull's eye image of spect. In Computers in Cardiology 2000. Computers in Cardiology, Boston: IEEE Press, 2000; 317-320.

[2] Haraguchi R, Sugimoto N, Eiho S, Ishida Y. Registration and superimposed display of coronary arterial tree on bull's eye map of spect. IEICE Transactions of Information and Systems January 2002;E85-D(1):69-76.

[3] Svane B, Bone D, Holmgren A, Landou C. Polar presentation of coronary angiography and thallium-201 single photon emission computed tomography. Acta Radiologica 1989; 30(6):561-574.

[4] Kim HC, Min BG, Lee TS, Lee SJ, Lee CW, Park JH, Han MC. Three-dimensional digintal subtraction angiography. IEEE Transactions on Medical Imaging October 1982;MI1(2):152-158.

Address for correspondence:

Ryo Haraguchi

Graduate School of Informatics, Kyoto University

Gokasho, Uji-City, Kyoto, 611-0011, Japan

haraguch@image.kuass.kyoto-u.ac.jp 